$$
\text { "tmcs-sipos" — 2009/11/1 — 17:36 — page 259 — \#1 }
$$

\title{
Teaching geometry using computer visualizations
}

\author{
ElviRA Ripco Sipos
}

\begin{abstract}
In this work we study the development of students' creativity using computeraided-teaching during IT classroom. Teaching geometry in Bolyai Grammar School specialized natural science classes is not an easy task. Here is introduced a new didactic means of teaching geometry which nevertheless requires the same effort to understand the material, but uses a different more active method to familiarize students with the topics. Traditional methods, and the use of compasses and rulers are not omitted either, as they develop the students' motor skills.
\end{abstract}

Key words and phrases: technological tools, computer software, teaching methods, classroom techniques.

ZDM Subject Classification: G60, G90, G05, G06, C39, U70, G43.

\section{Introduction}

Teaching mathematics in a secondary school is a very difficult task, its most interesting area being geometry built axiomatically. Helping students to understand and learn new theorems in geometry is an exciting task from a teachers point of view. Some psychological and didactical surveys show that the acquisition and learning of geometry in the secondary school is a very difficult task. This could be due to a lack of problem-solving-thinking or to the decreasing creativity of the students. Consequently few schoolchildren get to know the amazing world of the science of the Ancient Greek geometers'. The incompleteness and imperfectness of representations on paper and limited time available hinder the learning of axiomatically-based theoretical geometry. A great number of geometrical figures,

Copyright (c) 2009 by University of Debrecen 


$$
\text { "tmcs-sipos" — 2009/11/1 — 17:36 — page } 260 \text { - \#2 }
$$

their symmetries, perfectness and variety remain unknown for some students who have finished secondary school. One of the most important tools for children in the XXI century is their companion, the computer. Applying the computer in the teaching and learning processes plays an important role. It is important how we introduce the computer during the course of teaching and learning from the aspect of the achievement of the cognitive processes. The appearance and rapid development of dynamic geometry systems (DGS) has brought rapid progress in the teaching of geometry. My research aim is to map out in which proportions it is necessary and sufficient to apply it, since visualization on the computer is not the only teaching-device we have at our disposal to use during geometry lessons. Besides understanding the theorems, our students should find good proofs and it is necessary to practice the elementary geometrical constructions using compass and rulers. It is important to find an optimal qualitative and quantitative balance during lessons of time spent on the visualization and theoretical proofing of constructions.

I have been teaching Geometry 1 at Bolyai Grammar school for gifted pupils specializing in mathematics and natural sciences for more than four years in Senta. They have 144 geometry lessons per year and I teach every 4th lesson; that is 25 percent, all of which are held in the IT classroom. Some 75 percent of the lessons are "classical" geometry, using paper in "classical" constructions using pencil, rulers and compasses, because they have to develop their motor skills.

My method is: every student has a desk and a computer; pictures of the main/teacher's computer are reflected on the whiteboard, so that the students can follow the instructions. They can recognize new aspects using DGS. They are able to find new methods of proving theorems; they gain a visual perception of the properties of the given problem by "moving" the basic points on the drawing. This kind of imaging is impossible in classical constructions in geometry, because it would entail drawing hundreds of accurate, precise constructions on paper. I expect the students to be able to visualize mathematical and geometrical problems and achieve the problem-solving abilities at the end of the course. This teaching is unique in Serbia because the language of learning is Hungarian, and we are able to teach our students according to the plans of the special mathematical grammar schools in Belgrade, Novi Sad, Kragujevac and Nis. Considering that my students needed help in learning Geometry, and as I have read and studied some papers of eminent Hungarian and American researchers I have found that (as David Tall says in his book):
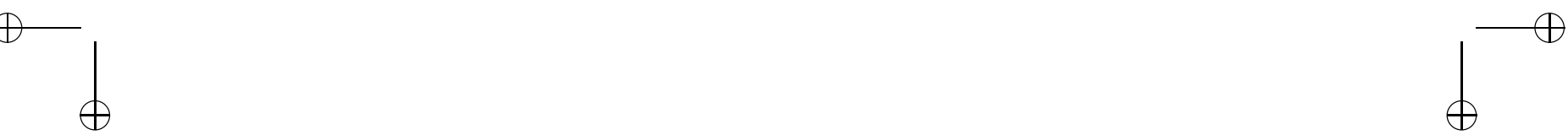
"tmcs-sipos" — 2009/11/1 — 17:36 — page 261 — \#3

Although the experts in mathematics may claim to share a coherent notion of proof, the cognitive development of proof is dependent on the cognitive structure and representations available to the learner at a given time. The formal concept of proof in terms of definition and logical deduction has a significant cognitive difficulty; it requires a reversal from "concepts described verbally" to "verbal definitions which prescribe concepts". This is likely to be highly confusing to non-experts. [13]

And in the study [14] he also writes:

By using suitable visual interpretations of mathematics it may be possible to draw one or more pictures which in total are formally generative, in the sense that they may be interpreted appropriately by some students to lead to corresponding formal arguments. For some students (successful natural learners), the pictures may allow them to construct a personal meaning for the definitions which allows them to build a rich conceptual structure to support the formal mathematics. For others (successful formal learners), working by interiorizing the definitions and reflecting on the formal proofs may also lead to successful under- standing of theorems and proofs, although further cognitive reconstruction is likely to be needed if the student wishes to integrate these new formal ideas with older intuitions.

The use of computers in teaching is based on some aspects of David Tall [15]:

The introduction of computer technology brings a new refinement to this theory. Whereas mode 1 is seen as the individual acting on and experimenting with materials that are largely passive, a computer environment can be designed to re-act to the actions of the individual in a predictable way. This new form of interaction extends Skemp's theory to four modes (Tall 1989) where building and testing environments are:

- Inanimate: the stimuli come from real objects which the individual may also be able to manipulate,

- Cybernetic: the stimuli come from systems which are set up to react according to pre-ordained rules,

- Interpersonal: the stimuli come from other people,

- Personal: the stimuli are from the individual's own cognitive structure.

The new cybernetic mode of building and testing concepts accords rich possibilities for the learning of mathematics. 
This is why I decided to institute computer-aided visualization in the teaching and learning of geometry in our school. The Simsone-line of the triangle with animation is one of the examples in our work:

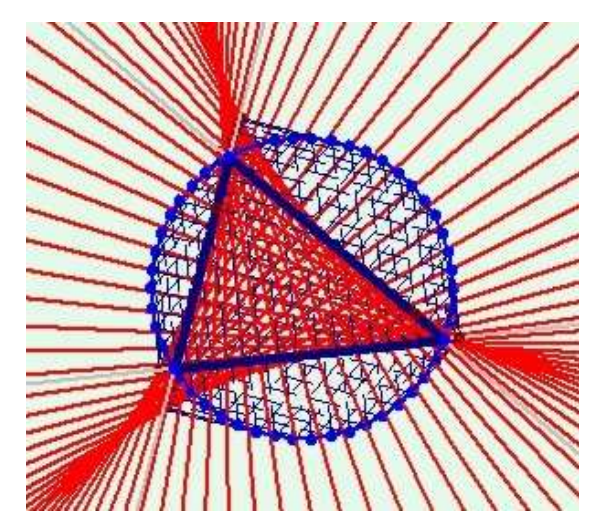

This visualization helps to:

- develop/improve spatial and perception skills;

- increase the intuitive talents;

- predict the theorems, and the properties of geometrical figures;

- increase divergent thinking and the checking of new ideas;

- recognize the "visible" proofs;

- motivate the students' activity;

- increase the students' enthusiasm.

The disadvantages of computer aided teaching are:

- a decrease of the proving claim;

- the deficiency of mathematical rigorousness, "since everything is visible on the drawing";

- that some students find the computer is inflexible and hence they become frustrated;

- it is still expensive for schools.

There is a 90-minute-long written exam in the school every three months, which contains tasks that have been taught in the previous three months, i.e. - problems to solve and prove. The chosen tasks are from our course book [6], which was translated into Hungarian by me. There are students from all over Vojvodina in our school, with different backgrounds, schools and teachers. But all of them are 
talented at mathematics, as all of them have passed the entrance exam and been accepted into this special grammar school to be taught through the Hungarian language, which is unique in Serbia. In Schoenfeld's [10] we find the four categories of mathematical behavior:

- Resources are the body of knowledge that an individual is capable of bringing to bear in a particular mathematical situation. Our students come from many different cities and settlements in Vojvodina. Some of them learnt mathematics in Serbian. They had courses that prepared them for our entrance exam, but their primary knowledge is very different. They usually need differential preparation and teaching to develop.

- Heuristics are rules of thumb for effective problem solving. They are fairly broad strategies for making progress on unfamiliar or difficult problems. Visualization helps students to find and investigate special cases, to exploit analogies, to explore different properties and to "work backwards". The first generations could use only their own imaginations and drawings on blackboard and paper. New generations gained experience in the visual perception of exploration. Observing specific cases, such as equilateral, isosceles and right triangles, or choosing one of the elements of a figure as a special value (after which the others are easily calculated), then one can spilt the given problem into subgoals, each of which is an easier but related problem, thus leading to the complex solution. Subsequently one can formulate a conjecture of the theorem, and can prove it. The straightforward solution of a Polya's geometry problem by means of an easier, related problem calls for (1) knowing how to use the right strategy; (2) knowing the appropriate strategy-versions for that problem; (3) generating appropriate, easier, related problems; (4) assessing the likelihood of being able to solve and exploit each of the easier problems; (5) choosing the right one; (6) solving the chosen problem; (7) exploiting its solution. [8]

- Control deals with the question of resource management and allocation during problem-solving attempts. The old group, before I began using visualization, could not be sure in the accuracy of their solutions in every case, because drawings on paper were not sufficiently precise, despite their accurate work. It was easier to measure some elements and to construct difficult figures using DGS. The new group tried to move basic elements on the computer's construction and was able to find out how these movings, transformations change the given figure. 
- Belief systems are one's mathematical world view, the perspective with which one approaches mathematics and mathematical tasks. The old group didn't have visualization; their imagination was the only thing they could use in solving the problems. The new group learned how to analyze some problems, and find a "tool" that helped them understand the unfamiliar questions, because each problem can serve as archetype for a large class of problems solved by similar techniques, although the key to each is different.

\section{Learning the mathematical concept by SKEMP}

In our everyday lives a large part of the knowledge we use is acquired directly from our environment and is not so abstract. The special difficulty of Mathematics and its power resides in its substantial abstraction and universality. Although the basic principles of teaching Mathematics are simple, knowing them is not important to the 'recipient' in the communication system (the student), but more necessary for the 'sender' (the teacher, who gives the mathematical concepts). The first basic principle is: We can't give concepts to anybody with definition of higher order of his/her knowledge, but if we show many suitable examples, one can understand the essence of the concept. The second basic principle is: We must be sure that the student still knows basic essences of the new example. Geometry looks like an environment where it is worth examining the visualization and the symbols, because drawings have a great significance. These symbols have a more abstract meaning than the visual drawings of some objects. The difference between the two kinds of symbols - the picture and the word - is that the picture looks like the given object, but the word doesn't have to be the same. In summary, the opposite characteristics and the characteristics complementing each other [11] are:

\begin{tabular}{|c|c|}
\hline Visual & Verbal-algebraic \\
\hline abstract space characteristics & abstract properties independent from the space \\
difficult to explain with words & easy to explain with words \\
demonstrates individual thinking & demonstrates socialized thinking \\
integrated, shows the structure & analytical, shows the details \\
simultaneous & one after the other (one-by-one) \\
intuitive & logical \\
\hline
\end{tabular}




$$
\text { "tmcs-sipos" — 2009/11/1 — 17:36 — page 265 — \#7 }
$$

The child's mathematical thinking means many things: it is partly a contact with an adult, a solo-activity with its own problems and strategies for its solution, or an informal idea of his/her own intuitions. He/she sees mathematics as an arbitrary series of actions, a set of tricks planned as an obstacle. That is why we the teachers have to examine the approach to the central problem of the cognitive and educational process.

\section{Introduction into the different types of geometry}

As Munkácsy Katalin writes in [7]:

Students should know that Geometry (and generally Mathematics) is not a natural science in classical terms. They have to know that we can't guarantee that mathematical theorems which have been proven are surely true, but still, they have been working well in past millenniums.

During the time of introduction and study of Hilbert's axioms, we become acquainted with the fact that there are 21 assumptions, which underline the geometry published in Hilbert's classic text "Grundlagen der Geometrie" [3]: the incidence axioms, ordering axioms, congruence axioms, continuity axioms and the single parallel axiom equivalent to Euclid's fifth (parallel) postulate, as we can read in Euclid's "Elements" [2]. But it is our mission - the teacher's duty today - to show the different possibilities of this axiom of parallels. At this point, the three basic axioms are presented: Playfair's, Lobachevsky-Bolyai's and Riemann's axiom of parallel lines. There are three classes of constant curvature geometries in three dimensions. All are based on the first four of Euclid's postulates, but each uses its own version of the parallel postulate. The "flat" geometry of everyday intuition is called Euclidean geometry (or parabolic geometry), and non-Euclidean geometry is called hyperbolic geometry (or Lobachevsky-Bolyai-Gauss geometry in [1]) and elliptic geometry (or Riemannian geometry). Spherical geometry is a non-Euclidean two-dimensional geometry. We use "Bolyai.exe" software written by Szilassi Lajos, [12], for experimenting with hyperbolic geometry, where students can understand the concept of the "line" and find out to their great surprise, two or more parallels to the given "line". The concept of infinity, that the boundary of the main circle in the Poincaré-model is not in "our world", is very important. 


$$
\text { "tmcs-sipos" — 2009/11/1 — 17:36 — page } 266 \text { - \#8 }
$$

Students usually construct some triangles in this Poincar'e-model, to find a sum of the angles of the triangle and count the defect of the triangle. So they understand that the axiom of Lobachevsky-Bolyai is equivalent, that the sum of the angles in that triangle is less than a straight angle. The special case of the triangle with zero sums of the angles is always found to be amazing. I always take a tour to astronomy and tell them the true story about Albert Einstein's "Theory of General Relativity", that in May, 1919, a team led by a British astronomer, Arthur Stanley Eddington claimed to have confirmed Einstein's prediction of gravitational deflection of starlight by the Sun while photographing a solar eclipse in Sobral, northern Brazil. [4]:

The perihelion precession of Mercury was the first evidence supporting the fact that general relativity is correct. Sir Arthur Stanley Eddington's 1919 expedition in which he confirmed Einstein's prediction for the deflection of light by the Sun helped to affirm the status of general relativity as a likely true theory. Since then, many observations have confirmed the correctness of general relativity. These include studies of binary pulsars, observations of radio signals passing the limb of the Sun, and even the GPS system.

I show them a picture from the report of Sir Arthur Eddington on the expedition to verify Albert Einstein's prediction of the bending of light around the Sun, the great center of gravity. This is a real model of non-Euclidean geometry in nature.

Spherical geometry is a non-Euclidean, two-dimensional geometry. I use the Lénárt Sphere kit to study spherical geometry, [5]. Our school has seven sets of these Lénárt Sphere since the "Bolyai Farkas Foundation" donated them to us. This tool is very useful, and my student like it. These geometry construction materials allow primary and secondary school students to investigate spherical geometry using a real sphere and the spherical equivalents of a compass and straightedge. With these new tools, students can com- pare and contrast relationships on the sphere with the corresponding relationships of Euclidean geometry. Working with non-Euclidean geometry keeps students' thinking fresh and deepens their insights into planar geometry. Investigating spherical geometry requires students to think creatively. And hands-on work with the Lénárt Sphere is a great opportunity for students to increase their spatial visualization skills. Naturally, we mostly study the wonderful world of Euclidean plane geometry. 


$$
\text { "tmcs-sipos" — 2009/11/1 — 17:36 — page } 267 \text { — \#9 }
$$

\section{The task}

The given problem was Euler's line. The preliminary knowledge of the students contained the important points of the triangle, as the center of the circumcircle, orthocenter, center of the inscribe circle and the centroid, which were studied during two lessons. First, the definition was given, and we constructed it on the acute triangle. The next step was its proof, to show correctly the related theorem and properties. The third thing was the construction of that important point, special cases of the isosceles, equilateral and right-angled triangle. The homework was the case of the obtuse triangle. The students were asked to make a good construction of adequate accuracy. This homework was checked by me, and I pointed out incorrect details and imperfect constructions.

The examination contained three parts (phases): 1 . Visualization on the computer; 2. Comprehensive tasks on marking; 3. Revision after the winter vacation.

Until we got to the proof of the property of the Euler-line OTH, my students knew the basics of the vector algebra such as their addition and multiplying with real numbers, and we had proved the Hamilton-theorem:

$$
\overrightarrow{O B}+\overrightarrow{O C}+\overrightarrow{O A}=\overrightarrow{O H}
$$

as well as the vector equality:

$$
\overrightarrow{C H}=2 \cdot \overrightarrow{O C_{1}}
$$

if $C_{1}$ is the midpoint of the segment $B C$ in the triangle $A B C$.

\subsection{Visualization on computer}

After we had given definitions of important points of the triangle, (they had had preliminary knowledge about these mathematical concepts), we drew them using DGS. Students were requested to use different colors to mark these points, $S$ as the center of the inscribed circle, $O$ to the center of the circumcircle, $H$ as orthocenter, $T$ as the centroid of the triangle. The next step was the moving of basic points, the vertices of the given triangle. What happened to these important points? How did they move? Where were they in different cases? Check their positions! Students found the answers to these questions themselves. They recognized the isosceles triangle, the equilateral triangle (there is a function to measure edges and angles of the triangle), and the right angle triangle. They had 
a chance to examine the cases of acute and obtuse triangles. They were able to form a theorem about the position of these important points, as if the triangle's obtuse points $O$ and $H$ were on different sides of the triangle or outside it. Then I gave a little help: "Hide for a moment point $S$, the center of the inscribed circle of the triangle! Connect points $O$ and $H$ !"

Their answers came very fast: The centroid was on this line $O H$. At this moment, we were able to define Euler's line, as a line which contained the centroid, the orthocenter and the circumcenter of the triangle. They were satisfied (me, too!) and with great enthusiasm they made a few animated drawings. They measured the length of the segments $H T$ and $T O$, and they found equality:

$$
2 \cdot \overrightarrow{O T}=\overrightarrow{T H}
$$

This was the appearance of the joy of discovery: "Eureka". Here are some examples of their works:

- Student E.A. made his drawing of Euler-line very well, this example is on the acute triangle, where all important points are inside of the triangle.

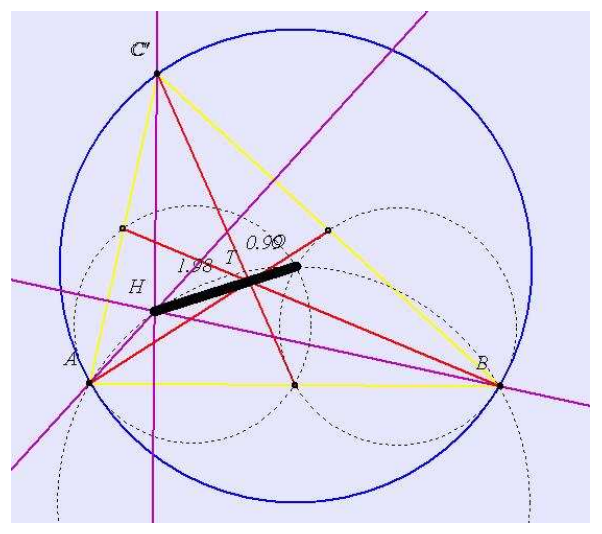

- Students B.A. and F.E. made their work on obtuse triangle, where one can see, that the orthocenter of the triangle and the circumcenter are on the opposite side of the triangle 
$\bigoplus$

$$
\text { "tmcs-sipos" — 2009/11/1 — 17:36 — page } 269 \text { — \#11 }
$$

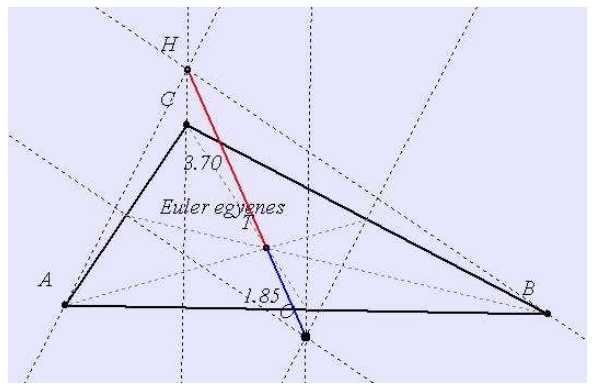

- Student T.S. drew the case of the right-angled triangle, where the orthocenter is the vertex of the right-angle, and the circumcenter is the midpoint of the hypotenuse.

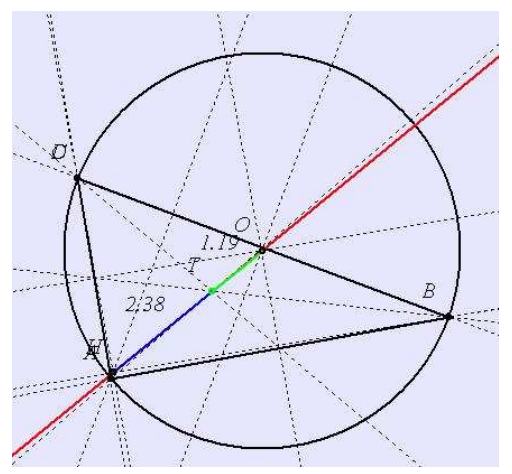

- Student N.A.E. drew a triangle without any signs, the important points are unknown, although well constructed.

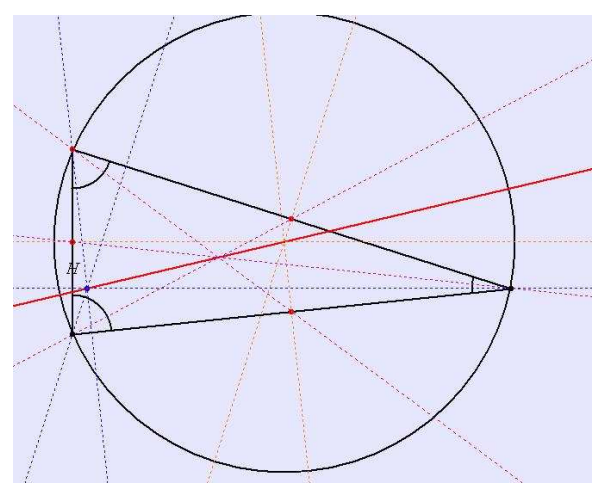


- Student N.L. drew this picture as the Euler-line. At the end of the lesson he was able to make only this one. But, next time, he recognized his mistakes, and made a new, better picture with our help.

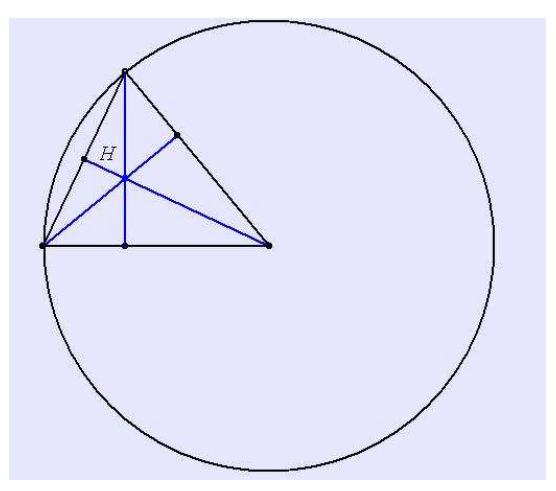

\subsection{Comprehensive tasks onto marking}

Comprehensive tasks onto marking were used to compare students' knowledge at this level of learning. There was a task to make a classical construction with compass and ruler. My aim was to develop their motor ability skills, to show the mistakes in drawing and to raise the level of their accuracy in the construction. Here are some examples of their works:

- Student H.Z. made a good construction, with appropriate signing, and using red for the important details. His only mistake was forgetting the capital letter in Euler's name. He indicated only Euler's line.

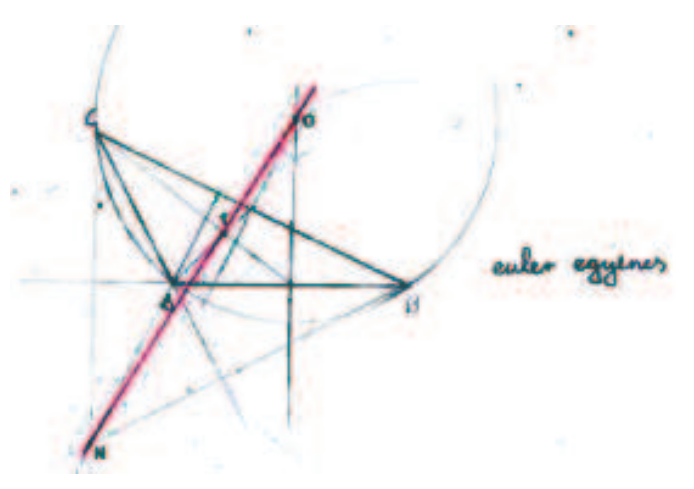


$\bigoplus$

$$
\text { "tmcs-sipos" — 2009/11/1 — 17:36 — page } 271 \text { — \#13 }
$$

- Student B.A. drew the Euler-line with the Euler-circle, showing nine points on it.

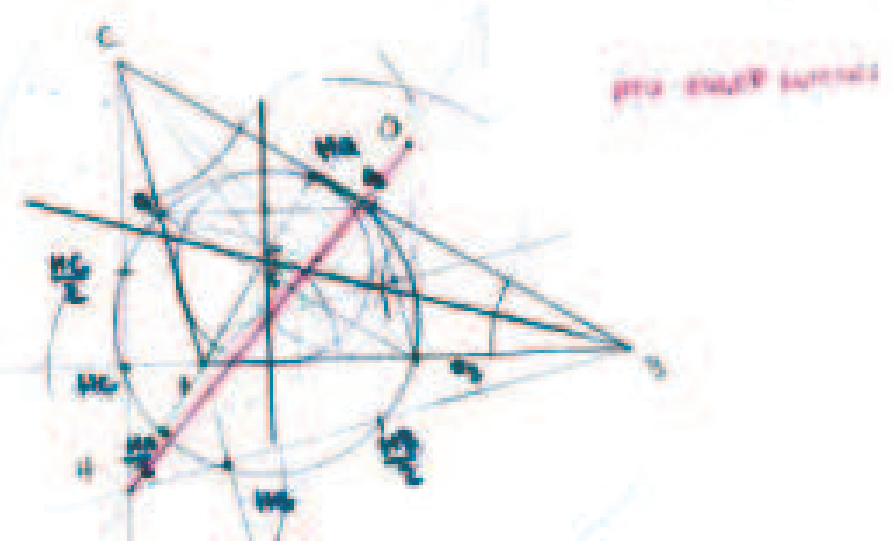

- Here is a very good construction from F.E

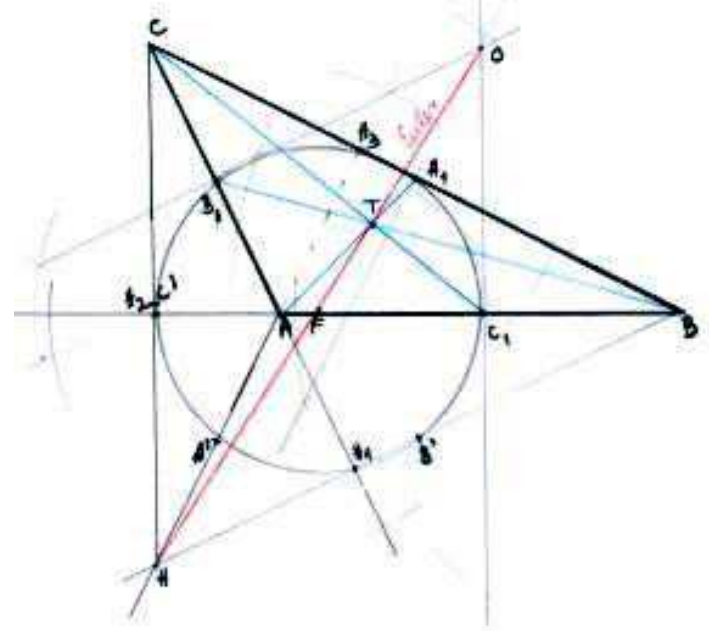

Furovock wör is Guler equars 
- Student V.I. has no accuracy yet, and he pays no attention to the constructions, but he has "mathematical brain". He is talented, demonstrated by what he provided during the last competitions.

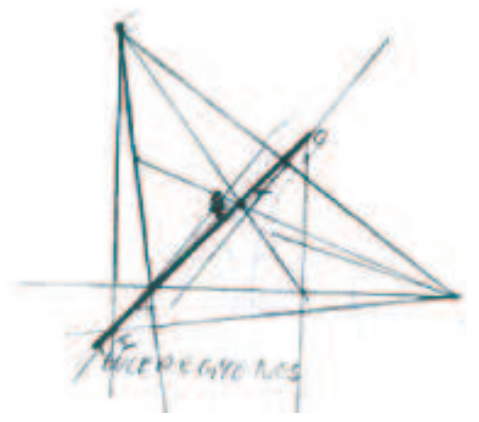

- Student L.I. has forgotten that this drawing had to be done with an obtuse triangle. The accuracy here is not important, although he had an adequate knowledge of the construction, shown beside the picture.
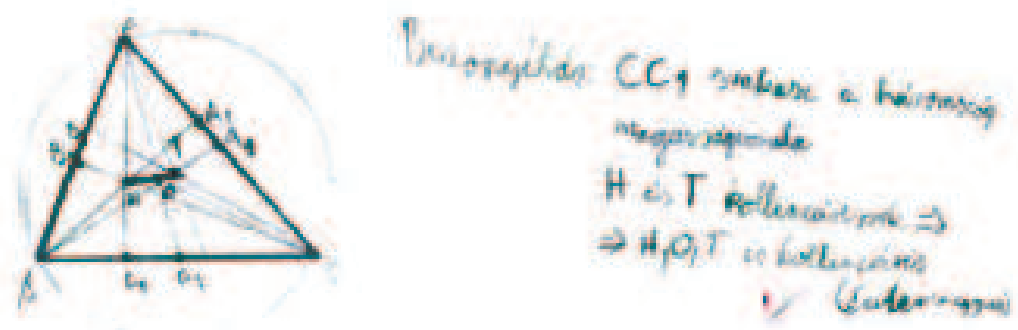

Translation: Proof: segment $C C_{1}$ is an altitude of the triangle $A B C$, points $H$ and $T$ (orthocenter and barycenter) are collinear points.

\subsection{Revision after the winter vacation}

Repetition after the winter vacation is always in our teaching plan to practice the theoretical proofing. It is because repetition is the mother of knowledge: "Repetitio est mater studiorum". Here are some examples:

- The best K.T. He is a little talent in mathematics. He works very hard. His solution was made on the basis of the similarity of the triangles $C T H$ and 
$T O C_{1}$. This is an excellent work and the nicest and simplest proof of the property of the Euler-line.
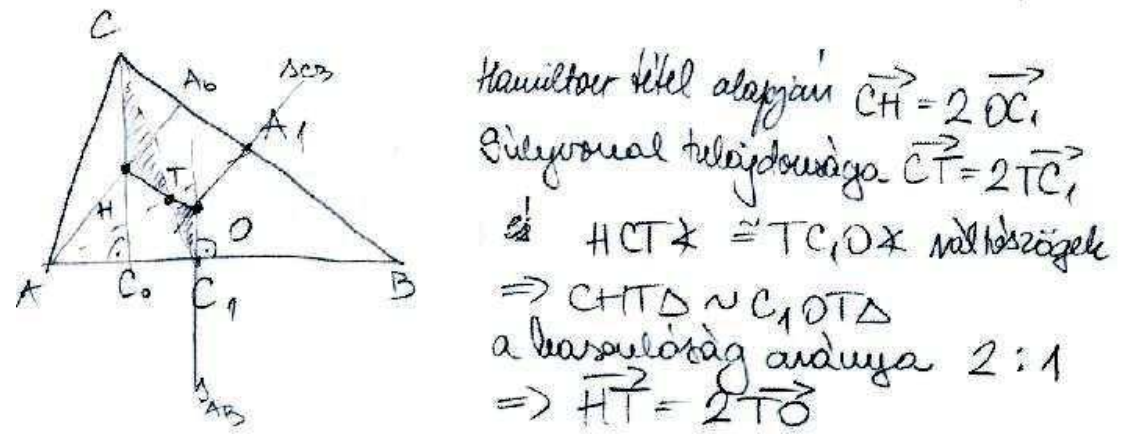

Translation: Based on the Hamilton-theorem:

$$
\overrightarrow{C H}=2 \overrightarrow{O C_{1}}
$$

Median line:

$$
\overrightarrow{C T}=2 \overrightarrow{T C_{1}}
$$

and angle $\mathrm{HCT}$ is equal to angle $\mathrm{TC}_{1} \mathrm{O}$ as alternate angles, that means triangles $C H T$ and $C_{1} O T$ are similar, ratio of similarity is $2: 1$,

that means

$$
\overrightarrow{H T}=2 \overrightarrow{T O}
$$

- A well done by B.D. He chose the proof by vectors that had been shown during the previous lessons, but his stage fright prevented him from doing it without error. He mistook one coefficient, although he knew the final result. Here, the vector equality was applied on the median with midpoint and the centroid, he used the property:

$$
\overrightarrow{O B}+\overrightarrow{O C}=2 \cdot \overrightarrow{O A_{1}}
$$

as well as the proportional division of the vector and the Hamilton-theorem, but he wrote sign $O$ as the center of the inscribed circle. It was only a little mistake in his work. 


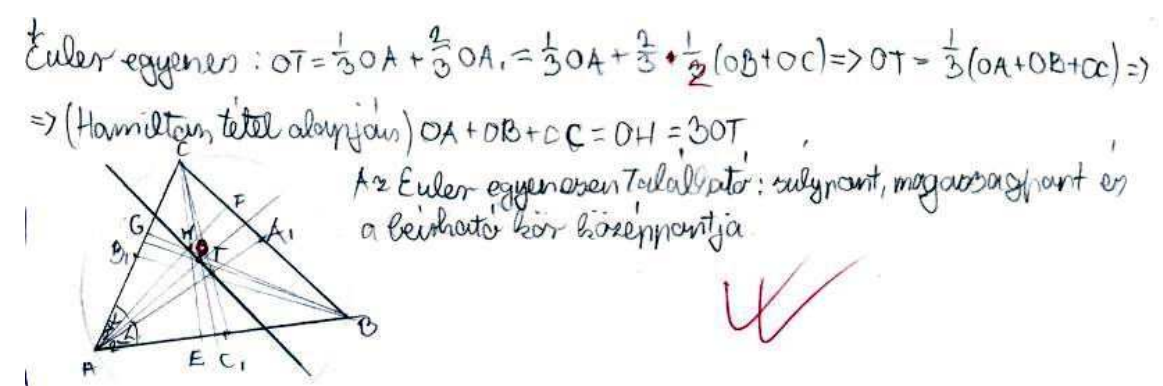

Translation: Points on the Euler's line: barycenter, orthocenter and the center of the inscribe circle

- A good V.A. She is one of our greatest "little mathematicians". Last year, she won second place on our school "Fekete Mihály" mathematics competition. She is very assiduous and conscientious which is why she prepared very hard for this examined work. She asked H.M., a student from the fourth grade, to help her in the preparations. H.M. has won every great mathematical competition in Serbia and some international competitions in mathematics, informatics and physics. He is a genius. He showed her his own solution using the scalar product of vectors, which concept was unknown to her. In his solution he used the feature according to which the two vectors are perpendicular to each other if their scalar product is equal to zero. Hence, she didn't learn these concepts she tried to remember it without understanding and the result was there.

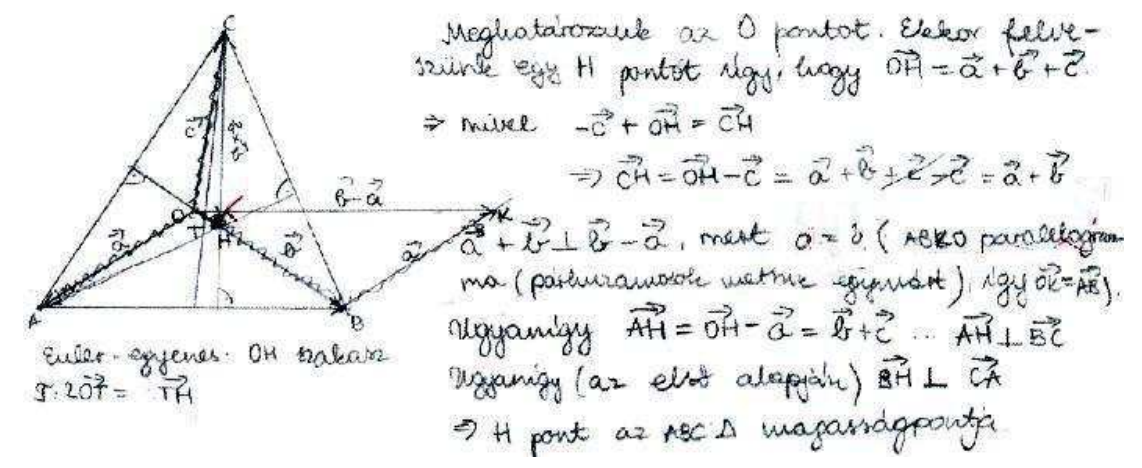

Translation: We construct (order) point $O$. Then construct point $H$ to be

$$
\overrightarrow{O H}=\vec{a}+\vec{b}+\vec{c}
$$


Because

$$
-\vec{c}+\overrightarrow{O H}=\overrightarrow{C H}
$$

that means

$$
\overrightarrow{C H}=\overrightarrow{O H}-\vec{c}=\vec{a}+\vec{b}
$$

Vector $\vec{a}+\vec{b}$ is perpendicular to vector $\vec{b}-\vec{a}$, because $a=b$ (ABKO is $a$ parallelogram, intersection of parallels) that why

$$
\overrightarrow{O K}=\overrightarrow{A B}
$$

Same case: vector $A H$ is perpendicular to vector $B C$, similarly vector $B H$ is perpendicular to vector $C A$. Hence point $H$ is the orthocenter of the triangle $A B C$.

- A.T. who wanted to be good and applied vector equality, the property of the medians but with wrong points $O$ (circumcenter) and $S$ (center of the inscribed circle).

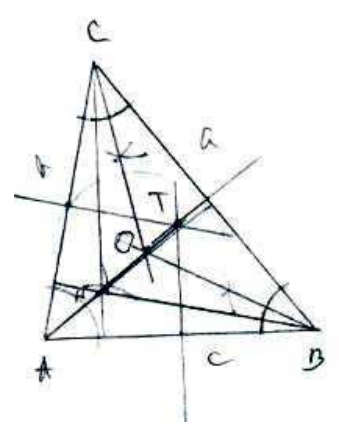

$$
\begin{aligned}
& \overrightarrow{C M}+\overrightarrow{A D} \\
& \overrightarrow{C M}=\overrightarrow{O M}-\overrightarrow{O C}=(\vec{a}+\vec{b}+\vec{c})-\vec{c}=\vec{a}+\vec{b} \\
& \overrightarrow{b-a}+\vec{b}+\vec{a} \text { ment a es la equenlc } \\
& \overrightarrow{O D}=\frac{\vec{a}+\vec{b}+\vec{c}}{3}=\overrightarrow{O M} \cdot \overrightarrow{0 j} \Rightarrow h
\end{aligned}
$$

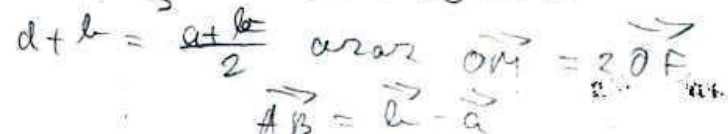

- There is a not so good N.L., too, who has forgotten almost everything during the winter vacation, didn't pay attention to the teacher's appeal at the revision.

\section{Plans for the future, continue}

The next task for the future is to continue this method into the second- and third-grades. In the second-grade there is trigonometry and the stereometry in the official plan. Trigonometry contains the tasks of proving the special theorems 


$$
\text { "tmcs-sipos" — 2009/11/1 — 17:36 — page 276 — \#18 }
$$

of Ceva, Menelaus, Ptolemy and Euler's line using trigonometrical tools,such as the sine- and cosine-theorems. Here we shall use Mathematica 6.2 software. In the third-grade there is analytical geometry, to describe a plane using coordinates, and equations. There are tasks to find and prove the above-mentioned theorems. Geogebra is very useful. The writer of this essay plans to follow the development of these students for four years and to test their learning of Euler's line in the fourth-grade, because I think that concepts learned from different methods will give the expected results.

\section{Results}

In the first phase, visualization helped in the exploration of new concepts, their properties, and to find and understand new theorems. In the second phase, students made a recall to the accurate construction made on computers by the dynamic geometry system. Their drawings were as accurate as possible. They made an effort to prepare as exact construction as possible using graphite pencil an A4 paper. The third phase took place one month later, after the assimilation period. There was an emphasis on theoretical proof. It was enough to remind them of Euler's line, and they knew what their task was: from the given triangle, across the important points of the triangle how one can get to the line OHT. But not all the solutions were adequate (to my disappointment). The nicest solutions were different in using geometrical tools, one using isometric transformation, and another using perspective transformation in their proof, the third student recalling the proof with vectors.

\section{References}

[1] J. Bolyai, Scientiam Spatii, Polygon, Szeged, 2002.

[2] Euclid, Elements, Gondolat, Budapest, 1983.

[3] D. Hilbert, Grundlagen der Geometrie, Osnovi geometrije, Matematicki Institut, Beograd, 1957.

[4] History of General Relativity, 07.10.2008, www.en.wikipedia.org.

[5] I. Lénárt, Nem-euklideszi kalandok, Key Curriculum Press, 2009.

[6] M. Mitrović, S. Ognjanović, M. Veljković, L. Petković and N. Lazarević, Geometrija I za matematičke gimnazije, Krug Beograd, 2003.

[7] K. Munkácsy, A matematikai bizonyításfogalom változásának hatása a tanításra, http://tandem. lauder.hu/ujsag/cikkek/20000209.html. 
[8] Gy. Pólya, A gondolkodás iskolája, Bibliotheca Kiadó, Budapest, 1957.

[9] Gy. Pólya, Mathematical Discovery on understanding, learning and teaching problem solving, John Wiley and Sons Inc., New York, 1962.

[10] A. H. Schoenfeld, Mathematical Problem Solving, Academic Press, INC., New York, 1985.

[11] R. R. Skemp, The Psychology of Learning Mathematics, Hungarian Translation (C)1975 Klein Sándor, Lawrence Erlbaum Associates, New Jersey, U.S.A, 42-44, $138,152$.

[12] L. Szilasi, 05.06.2009, http://www.jgytf.u-szeged.hu/tanszek/matematika/Bolyai/index.html.

[13] D. Tall, The Cognitive Development of Proof: Is Mathematical proof For All or For Some?, Conference of the University of Chicago Scholl Mathematics Project, August, 1998.

[14] D. Tall, Computers and the Link Between Intuition and Formalism, in: Proceedings of the Annual International Conference on technoogy in Collegiate Mathematics, Addson-Wesley Longman, 417-421.

[15] D. Tall, Advanced Mathematical Thinking and The Computer, in: Proceedings of the 20th University Mathematics Teaching Conference, Shell Centre, Nottingham, 1996, 1-8.

[16] D. Tall, The Psychology of Advanced Mathematical Thinking: Biological Brain and Mathematical Mind, Conference of the International group for the Psychology of Mathematics Education, Lisbon, July, 1994.

ELVIRA RIPCO SIPOS

BOLYAI GRAMMAR SCHOOL

SENTA

E-mail: siposelvira@bolyai-zenta.edu.rs

(Received February, 2009) 